\title{
NEW POSSIBILITIES \\ OF SWIFT HEAVY ION IMPLANTATION IN MATERIAL SCIENCE AND TECHNOLOGY
}

\author{
B. SLOWIŃSKI ${ }^{a, b *}$ \\ anstitute of Physics, Warsaw University of Technology \\ Koszykowa 75, 00-660 Warsaw, Poland \\ ${ }^{b}$ Institute of Atomic Energy, Otwock-Swierk, Poland
}

\begin{abstract}
We summarise briefly the advantages of swift heavy ions $(\approx 1 \mathrm{MeV} / \mathrm{u})$ application to analysis and treating of solids in order to modify their properties. As an illustration some examples of this application are quoted.
\end{abstract}

PACS numbers: $61.80 .-\mathrm{x}, 61.80 . \mathrm{Jh}$

\section{Introduction}

Until quite recently the notion of ion implantation meant a procedure of treating solids with beams of ions at energy in the interval between $\approx 10 \mathrm{keV}$ and $\approx 500 \mathrm{keV}$ in order to achieve desirable changes of their physical, chemical, and technical properties. Because such ions do not penetrate deeply into a substratum, ranging from $\approx 0.01 \mu \mathrm{m}$ to $\approx 1 \mu \mathrm{m}$, this way one can create in the irradiated amorphous material a thin planar coating nearby surface with modified chemical behaviour in different active media altering, as a rule, the chemical reaction rates, as well as mechanical and physical characteristics. Of special importance remains the application of ion implantation in semiconductor technology which actually turned out to be practically a unique tool for fabrication an enormous diversity of sophisticated microelectronic devices. Nevertheless, such low energy ion implantation is far from exhausting all potential possibilities inherent in ion beams irradiating materials. Indeed, another area of promising, useful and interesting applications of this time high energy heavy ions in radiation material physics and technology arose when more powerful accelerators of ionized particles of energy of $\approx 1 \mathrm{MeV} / \mathrm{u}$ and even of several $\mathrm{GeV}$ were used. As a consequence new physical phenomena appeared which, in turn, give new possibilities of their practical applications. With the help of the ions of $\approx 1 \mathrm{MeV} / \mathrm{u}$ energy (often called as swift heavy ions or simply SHI) one can solve a variety of important problems of

*e-mail: slowb@cx1.cyf.gov.pl 
practical and cognitive significance. Therefore, SHI having sufficiently large projected ranges in solids (more than $\approx 10 \mu \mathrm{m}$ ) as compared with the thickness of the so-called technical surface layer $(\approx 3 \mu \mathrm{m})$ can create buried layers in a material with quite different properties (electrical, optical, thermal, chemical etc.) than the substrate medium. They are also commonly applied for modelling an influence of neutrons on different materials used in nuclear plants and exposed to radiation because this way the time of accumulation of required doses of damages in these materials is shorter by a factor of $10^{3}-10^{6}$ in comparison with the time of exposition to neutron beams [3]. Moreover, the samples irradiated with heavy ions are significantly less radioactive. Another way of wide application of energetic ions is for the production of spatial structures in semiconductors and for the creation of nuclear filters.

The article summarises briefly the advantages of swift heavy ions $(\approx 1 \mathrm{MeV} / \mathrm{u})$ application in order to modify the properties of different materials. (For more detailed discussion of other aspects of ion implantation techniques we refer the reader to monographs [1] and review articles [2].) Several typical examples illustrating this application, except for the above mentioned and commonly known ones, are also discussed in brief: (1) modification of temperature dependence of manganin resistance improving its characteristics as a pressure sensor $[6,7]$, (2) increase in diffusion through latent tracks [8], (3) investigation of defect evolution in nanocrystalline $\mathrm{Pb}$ and $\mathrm{ZrO}_{2}$ with different initial grain sizes from 10 to $300 \mathrm{~nm}[9]$.

\section{New possibilities of swift heavy ions application}

Except for some similarities, SHI, penetrating into amorphous materials, reveal many remarkable differences in comparison with the ions of traditional implantation energy range. Below we summarize in short the most important of them:

1. Projected ranges of such ions in irradiated materials exceed $\approx 10 \mu \mathrm{m}$ and so make possible to avoid an influence of surface effects of flowing down of point defects and, as a consequence, to consider the relevant implanted layers as macroscopic buried metastable structures. Therefore, for example, the projected range of ${ }^{12} \mathrm{C}^{2+}$ ions of energy of $8.25 \mathrm{MeV} / \mathrm{u}$ in iron and silicon are equal to 70.1 and $185.0 \mu \mathrm{m}$ respectively, whereas the similar ranges for ${ }^{84} \mathrm{Kr}^{7+}$ equal 11.1 and $27.4 \mu \mathrm{m}$.

2. New created buried layers have, as a rule, different electric, optical, thermal, chemical etc. properties than substrate materials.

3. As a result of intense enough energy deposition along the ion trajectory and in an implanted layer the temperature around the individual ion track may exceed the melting temperature of the substratum material and the process of (re)crystallisation may there occur much easier creating this way required spatial configurations having expected properties as well as quasi-macroscopic channels allowing to penetrate outside and to stimulate the process of purification. Figure 1 illustrates such a phenomenon for diamond exposed to ${ }^{129} \mathrm{Xe}$ ions of energy equal to $124 \mathrm{MeV}[3,4]$. 


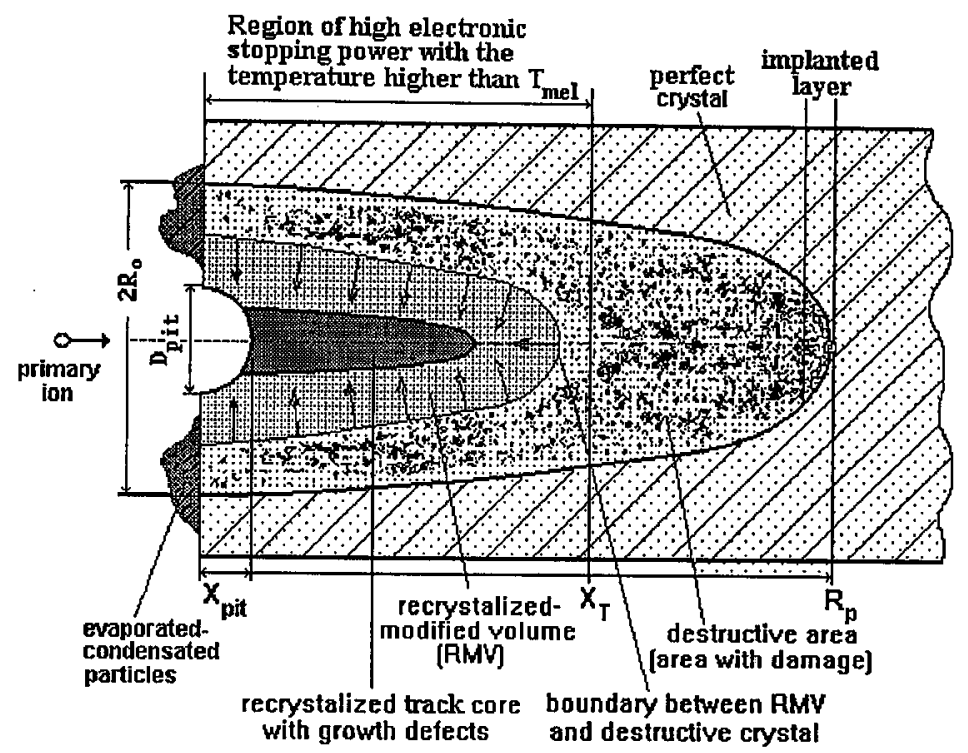

Fig. 1. Implantation of $124 \mathrm{MeV}$ energy ${ }^{129} \mathrm{Xe}$ ions into diamond. $D_{\text {pit }} \approx 3 \mu \mathrm{m}$ (after [4] with author's permission).

4. At high energies of ions their inelastic ionisation losses exceed the elastic ones, i.e. $(\mathrm{d} E / \mathrm{d} x)_{\text {inel }}>(\mathrm{d} E / \mathrm{d} x)_{\mathrm{el}}$, which changes the process of accumulation and evolution of defects during implantation, especially in the case of dielectric and semiconductor materials when the lifetime of local overheating around "tracks" may turn out to be sufficient in order to stimulate processes of diffusion and recrystallization.

5. For some problems high-energy ions proved to be a unique tool, for example, for modelling a spectrum of cosmic rays which produce damages in electronic devices and living tissues exposed to high energy radiation in different space programmes of investigation [3].

6. Such ions cause considerably less damages of the surface layers of implanted solids than ions of much lower energy do.

7. The energy losses of heavy ions for excitation of electronic subsystems of a substrate material reach their maximum value at the energy of ions around $\approx 1 \mathrm{MeV} / \mathrm{u}$. A direct observation in situ of luminescence increased in such a way make possible to investigate in more detail the process of evolution of defected structure of irradiated materials [5].

\section{Main trends with swift ions}

Owing to the above listed advantages of swift heavy ions application one can outline briefly the main trends in the relevant investigations:

1. Modification of characteristics (electric, optical, thermal, chemical etc.) of materials themselves (i.e. not only their surfaces).

2. Change of diffusion processes in materials. 
3. Purification, passivation, hardening and radiation resistance increase in materials.

4. Modelling of some (physical, chemical, biological) properties of materials and complex structures, such as, for instance, microelectronic devices.

\section{Several examples}

Below we present some examples illustrating a usefulness of various application of swift heavy ions. Of course, they do not exhaust all the diversity of the use of deep ion implantation. For more information in the field we refer the reader to the current literature and, in particular, to [2-4] and, for example, to GSI Scientific Annual Reports.

\subsection{Modification of temperature dependence of manganin resistance}

Manganin is commonly used as a convenient pressure gauge within the large range of values above about $100 \mathrm{MPa}$ owing to the linear dependence of its electric resistance on pressure. Nevertheless, the manganin resistance reveals acceptable weak temperature dependence within too narrow temperature interval only which may cause some systematic errors, difficult to be taken into account. Therefore, attempts have been made to widen this interval using ion implantation techniques and some promising results were obtained so far at comparatively rather

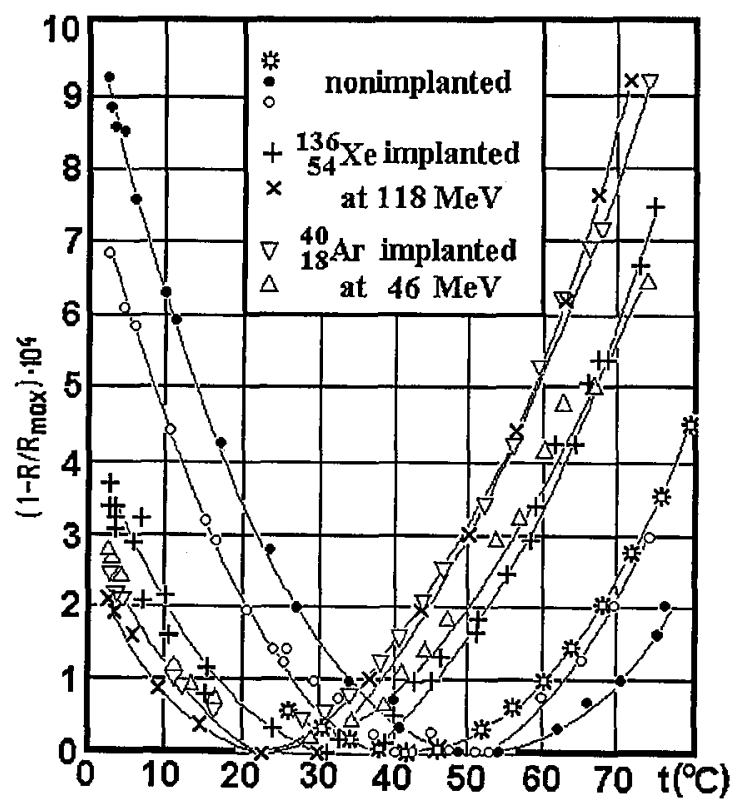

Fig. 2. Temperature dependence of the relative resistance of implanted and nonimplanted manganin slices $[6,7]$. 
low doses of $\mathrm{Ar}$ and $\mathrm{Xe}$ ions, equivalent to the implanted ion concentration of about $10^{18} \mathrm{~cm}^{-3}$ within the layers of thickness of 0.25 and $0.34 \mu \mathrm{m}$, respectively $[6,7]$. Figure 2 displays the difference in temperature dependence of the relative resistance of implanted and nonimplanted manganin foil $[6,7]$.

\subsection{Observation of diffusion through latent tracks}

In order to increase the diffusion constant $D_{\mathrm{Ar}}$ of argon gas through polymer foils the authors of [8] irradiated stack of Makrofol $K G$ foils, each consisting of 5 layers of $30 \mu \mathrm{m}$, with uranium ions of $11.4 \mathrm{MeV} / \mathrm{u}$, perpendicularly to the surface. The fluence was between $3 \times 10^{10}$ and $5 \times 10^{11} \mathrm{ions} / \mathrm{cm}^{2}$. The results of measurement of the diffusion of argon through all foils at $21^{\circ} \mathrm{C}$ is shown in Fig. 3 as a function of ion range $R$. Quoted are there the relevant calculation of energy loss of such ions vs. $R$. It is clearly seen from the figure that $D_{\mathrm{Ar}}$ rapidly increases with increasing $R$, at least up to $R \approx 100 \mu \mathrm{m}$.

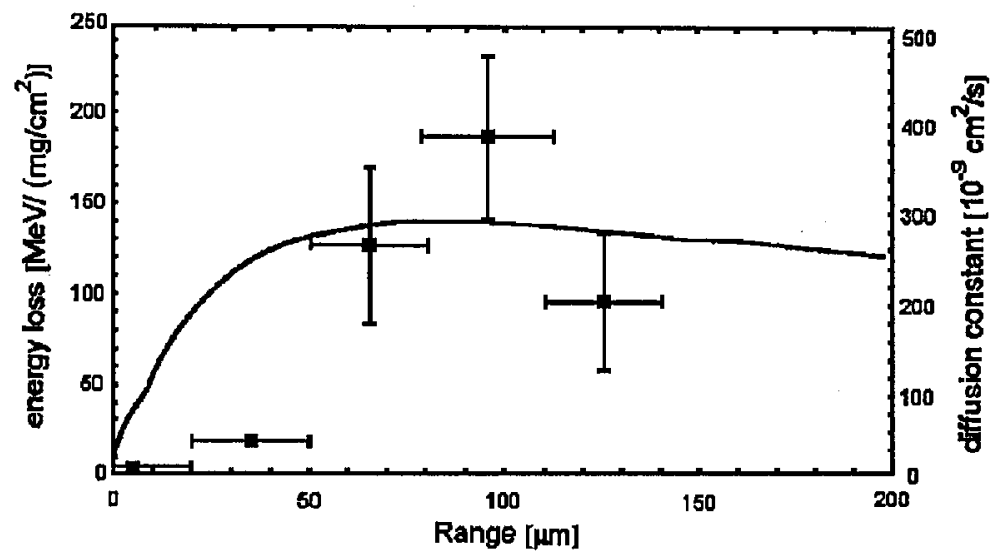

Fig. 3. The diffusion constants of one stack irradiated with $5 \times 10^{11}$ ions $/ \mathrm{cm}^{2}$ uranium ions. The theoretical energy loss by TRIM is shown by solid line (from [8] with authors' permission).

\subsection{Defect evolution in nanostructured materials}

In order to search for enhanced radiation resistance materials the authors of [8] have studied the correlation between defect density and the grain size of nanocrystalline $\mathrm{Pb}$ and $\mathrm{ZrO}_{2}$ with different initial grain sizes from 10 to $300 \mathrm{~nm}$. They irradiated these materials using $4 \mathrm{MeV} \mathrm{Kr}$ ions with fluences from $1 \times 10^{15}$ to $2 \times 10^{16} \mathrm{Kr}$ ions $/ \mathrm{cm}^{2}$. The results for $\mathrm{ZrO}_{2}$ show that at about $100 \mathrm{~nm}$ of grain size a saturation occurs in the defect density for $\mathrm{ZrO}_{2}$ [9]. Similar investigation for nanocrystalline $\mathrm{Pb}$ demonstrated a quite linear dependence between defect density and grain size [8]. The conclusion of the authors of [8] is that both nanocrystalline $\mathrm{Pb}$ and $\mathrm{ZrO}_{2}$ show enhanced radiation resistance and are suitable materials for applications in irradiation environments and space research [8]. 


\section{Concluding remarks}

Numerous applications of swift heavy ions of practically all the periodic table implanted into a large variety of materials involves several problems and needs. In the first place there is the further investigation, both theoretical and experimental, of stopping power of different ions (swift and slow, light and heavy, bare and screened) in miscellaneous targets (elemental and compound, amorphous and crystalline or polycrystalline, insulators and conductors). Furthermore, of particular importance mainly from the practical point of view is also the search for scaling (or similarity) properties which may occur in the process of ion implantation, for example, the similarity of stopping number in various media, as observed in the case of protons [10], as well as analogous feature of implanted profiles (energy, defect and mass deposition). The relevant findings, if successful, would permit to reduce considerably a number of necessary measurements of systems of target materials-implanted ions, which are of potential interest.

\section{References}

[1] Chr. Lehmann, Interaction of Radiation with Solids and Elementary Defects Production, North-Holland, Amsterdam 1977; J.S. Williams, J.M. Poate, in: Ion Implantation and Beam Processing, Academic Press, Sydney 1984.

[2] R.P. Sharma, Phys. News 7, 1 (1976); J.P. Biersack, Nucl. Instrum. Methods Phys. Res. B 35, 205 (1988); P. Sigmund, Nucl. Instrum. Methods Phys. Res. B 135, 1 (1998).

[3] Y.A. Didyk, D.Sc.Thesis, Dubna 1995.

[4] A.M. Adawi, D.Sc. Thesis, Dubna 1998.

[5] Y.A. Didyk, V.A. Skuratov, JINR Comm. 14-96-159, Dubna 1996.

[6] B. Słowiński, T. Wilczyńska, R. Wiśniewski, to be published in Nukleonika.

[7] B. Słowiński, R. Wiśniewski, JINR Comm. E-14-89-512, Dubna 1989.

[8] G.U. Bacmeister, F. Petersen, W. Enge, GSI Scientific Report 1994, p. 191.

[9] M. Rose, A.G. Balogh, H. Hahn, GSI Scientific Report 1996, p. 134.

[10] J. Lindhard, M. Scharff, Mat. Fys. Medd. Dan. Vid. Selsk. 27, 1 (1953). 